\title{
EuRegionale 2008: Drei + drei + fünf = eine Region?!
}

\author{
Die Bedeutung von Kommunikation nimmt bei grenzüberschreitenden Regional- \\ entwicklungsprojekten zu. Ein Beispiel ist das Drei-Länder-Land um Aachen, das \\ derzeit die EuRegionale 2008 vorbereitet. Aus dem Entwicklungsprozess des \\ Konzeptes lassen sich erste Schlussfolgerungen für ein grenzüberschreitendes \\ Regionalmanagement ableiten.
}

$\mathrm{D}$ Von Frauke Burgdorff und Ralf P. Meyer rei Sprachen, drei Nationalstaaten und fünf Kulturräume treffen im Drei-Länder-Land um Aachen aufeinander. Der Grundstein für diese Vielfalt wurde schon vor Karl dem Großen gelegt. Die wechselnde Vorherrschaft von Spaniern und Franzosen, Österreichern und Preußen sowie die schmerzhaften Einschnitte der beiden Weltkriege taten ihr übriges. Da kulturelle Traditionen in der Regel beharrlicher sind als politische Wechselspiele, haben sich die Sprach- und Kulturgrenzen relativ unabhängig von Schlagbäumen, Wällen und Zäunen entwickelt: So wird einerseits im unmittelbaren Grenzraum ein gemeinsamer Dialekt gesprochen, andererseits teilt sich die belgische Grenzregion in drei Sprach- und Kulturräume. Und schlieslich sind mit dem Zusammenwachsen Europas die Grenzen keine wahrnehmbaren Hürden mehr.

Das Grenzland könnte also ein gemeinsamer Entwicklungs- und Lebensraum sein, dessen naturräumliche, kulturelle, soziale und wirtschaftliche Potenziale genügend Anknüpfungspunkte und Anreize für eine trinational integrierte Entwicklung bieten. Dahinter verbirgt sich auch die Möglichkeit, von der jeweiligen räumlichen (und politischen) Randlage weiter ins Zentrum des Interesses zu rücken. Denn die Orientierung in Richtung der nationalen Zentren - der Randstad Holland auf niederländischer, dem ,Flämischen Diamanten" auf belgischer und dem Rhein-Ruhr-Gebiet auf deutscher Seite - sorgt immer wieder dafür, dass die unmittelbaren Nachbarn nur über Umwege von den Planungen und Entwicklungen des jeweils anderen in Kenntnis gesetzt werden.

Um diese Situation des ,Rücken an Rücken“ aufzubrechen und die jahrzehntelangen Bemühungen für eine bessere grenzüberschreitende Zusammenarbeit weiter zu unterstützen, hat die Aachener Region ihre niederländischen und bel- gischen Nachbarn bei der Bewerbung um die Ausrichtung der nordrhein-westfälischen REGIONALE 2008/10 von Anfang an mit ins Boot geholt. Bei diesem Wettbewerb geht es darum, innerhalb eines selbst gewählten konzeptionellen Rahmens Projekte, Ereignisse und Initiativen zu entwickeln, die das innere und äußere Profil der Region stärken und ihre naturräumliche, kulturelle, aber auch wirtschaftliche Entwicklung vorantreiben. Die Wettbewerbsunterlagen konnten nach eineinhalb Jahren intensiver Arbeit am 15. Oktober 2001 der nordrhein-westfälischen Landesregierung übergeben werden. Es handelt sich hierbei um ein Konzept, das entlang der Leitthemen FremdGehen (Kultur, Geschichte, Kommunikation und Kunst), StadtFinden (Siedlungs- und Landschaftsentwicklung) und GrenzWissen (Wissenschaft, Wirtschaft, Bildung, Arbeit) grenzüber- schreitende Projektkorridore und erste Projektideen darstellt, die der Region als Leitfaden für die weitere Entwicklung bis zum Präsentationsjahr 2008 dienen können. Dieses Konzept wurde mit Hilfe einer Vielzahl von Akteuren erarbeitet, die ihre Ideen und ihr Wissen aus den Teilregionen (Nationen) oder zu bestimmten Themenfeldern zur Verfügung gestellt haben. Der Konzeptentwicklung lag ein breit angelegter Kommunikationsprozess zugrunde, der sich der Herausforderung stellen musste, drei nationale Teilräume auf unterschiedlichen Ebenen einzubinden.

In naher Zukunft kommen zu diesen Kommunikationssträngen natürlich noch die entsprechenden EU-Gremien und vergleichbare Regionen in Europa hinzu. Denn das Ziel, Modellregion für Europa zu werden, kann nicht nur am eigenen Dreiländerpunkt erreicht werden.

\section{Der Kommunikationsprozess}

Das Zentrum der Kommunikationsarbeit bildete die Arbeitsgruppe EuRegionale 2008 (vgl. Abbildung 1), deren Mitglieder sich aus den Verwaltungen und Organisationen speisten, die den Bewerbungsprozess mit finanziert haben: acht Teilregionen aus dem Grenzraum, die Kammern und weitere intermediäre Einrichtungen.

Dieses Gremium hatte drei zentrale Funktionen: Die generellen Leitlinien der Bewerbung abzustimmen, das Wissen über die jeweilige Teilregion an die Partner zu kommunizieren und die zentralen Akteure vor Ort in den Prozess einzubinden. Eine eigens für den Bewerbungsprozess eingerichtete Geschäftsstel-

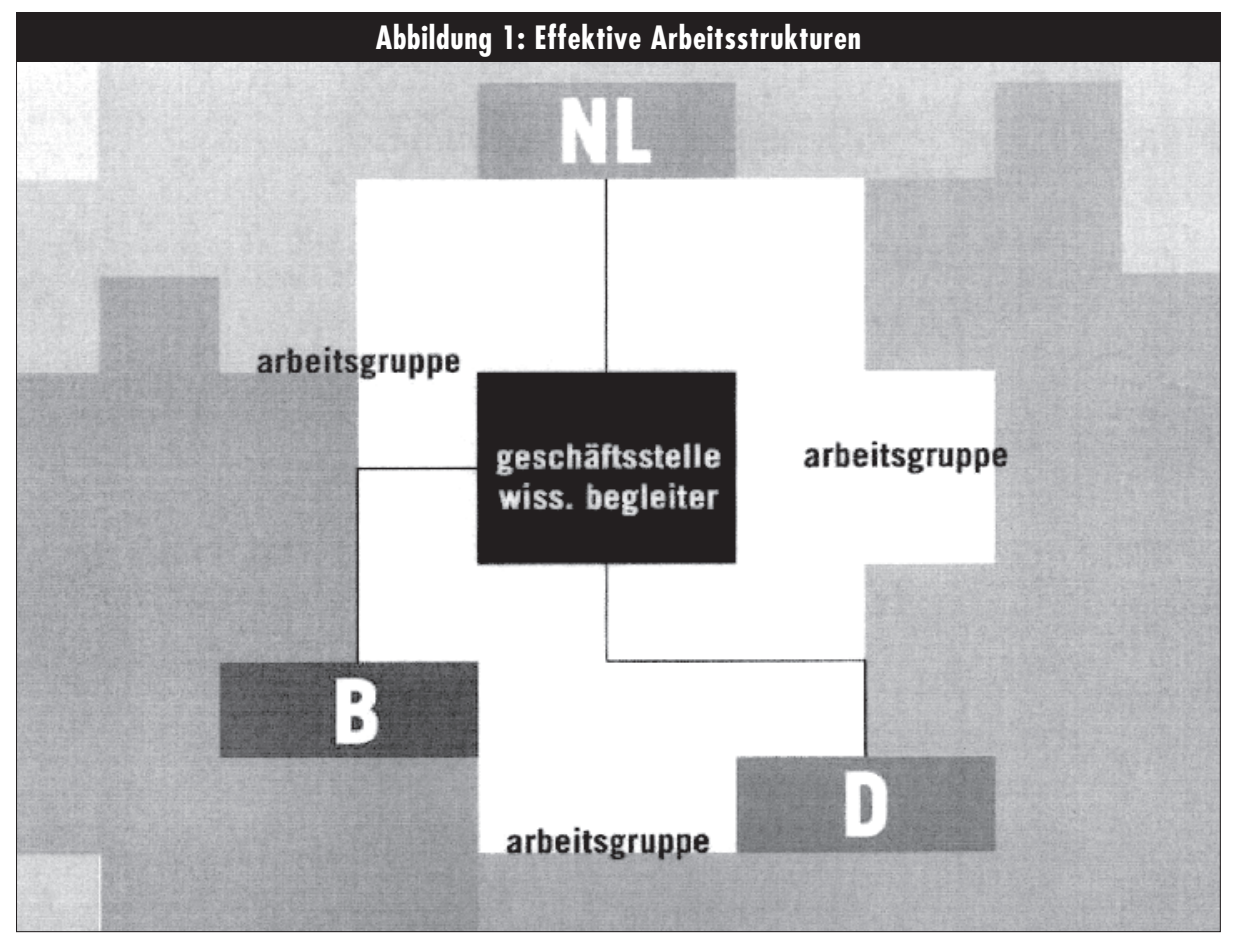


le hat die Arbeitsgruppe betreut und fungierte darïber hinaus als wichtigste Kommunikationsschnittstelle des Bewerbungsprozesses.

Zur Entwicklung des Konzeptes wurden in verschiedenen Phasen Kreativwerkstätten und Workshops durchgefïhrt. In der Anfangsphase (Auftaktworkshop) war es zunächst wichtig, die generelle Zielrichtung der Bewerbung zu definieren. In den Kreativwerkstätten haben Vertreter aller drei Nationen potenzielle Handlungsfelder, die zentralen Stärken und Schwächen der Region, die wichtigsten strukturellen Brüche, die aufgrund der Grenzen entstanden sind, aber auch erste Projektideen zusammengetragen. Die Teilnehmer waren nicht nur wichtige Ideengeber, sondern sollten auch Botschafter des EuRegionale 2008-Gedankens sein. Der sich anschließende Auswertungsworkshop fand in kleinerem, internem Kreise statt und diente dazu, das bislang themenbezogen Zusammengetragene interdisziplinär zu verknüpfen, zu filtern und der Arbeitsgruppe damit die weitere Weichenstellung zu erleichtern.

Zusätzlich zu diesem Ideen- und Kreativitätsschub aus der Region war es uns wichtig, auch den unvoreingenommenen, sachverständigen Blick von außen einzufangen, um Betriebsblindheit vorzubeugen und das bisher Erarbeitete kritisch zu reflektieren. Deshalb wurde an dieser Stelle des Verfahrens ein trinationales Expertengespräch mit Fachvertretern von Jugendsoziologie über Kunst und Architektur bis Wirtschaft aus allen drei Ländern geführt. Diese zweitägige Beratung war immens wichtig, um eingetretene Pfade zu verlassen und vor allem eine starke Rückendeckung für fachlich mutige Entscheidungen zu bekommen.

Nun galt es das bisher Erarbeitete wieder in die regionale Breite zu tragen und gemeinsam mit Fachvertretern aus der Region erste Projektideen zu diskutieren und sie durch das Engagement vor Ort zu bereichern. Einige dieser Konsultationsrunden haben sich als wichtige Ideengeber für die Projektfindung erwiesen, andere haben sich bereits heute mit ihren Ideen so weit verselbstständigt, dass sie ihre Projektideen eigenständig zur Antragsreife bringen werden.

\section{Kommunikation mit den BewohnerInnen}

Die einzelnen Ergebnisse des Entwicklungsprozesses wurden - um dem Projekt von Anfang an eine breite und tragfähige (eu)regionale Basis zu verleihen - fortlaufend an die allgemeine und politische Öffentlichkeit vermittelt. Die Herausforderung, ein komplexes Konzept anschaulich und be- wohnernah darzustellen, war dabei von Anfang an sehr groß und konnte nur durch ein breites Set an Kommunikationsinstrumenten bewältigt werden, die sowohl eine Vermittlungsaufgabe übernahmen als auch zum Mitmachen anregten. Bei der regionalen Verbreitung nahmen die Tageszeitungen vor Ort eine ganz zentrale Rolle ein. Die regelmäßige Berichterstattung über den laufenden Prozess, die Fokussierung auf konkrete grenzüberschreitende Projekte war wichtig, um die EuRegionale 2008 an die Frau bzw. an den Mann zu bringen.

Besondere Aufmerksamkeit erhielt darüber hinaus ein Fotowettbewerb, eine Wanderausstellung, die mit eigenen Eröffnungsveranstaltungen an acht Standorten in der Region zu sehen war und natïrlich die Internetseite (www.euregionale2008.de), auf der sich Interessierte informieren und an gleicher Stelle ihre eigenen Projektideen zu den einzelnen Leitthemen einbringen können. Knapp 50 Projektideen sind auf diesem Wege eingegangen und der Strom ist noch nicht versiegt!

\section{Blick zurück nach vorn}

Die EuRegionale 2008 steht eigentlich jetzt erst in den Startlöchern. Das Konzept ist nur vorläufig abgeschlossen, die Entscheidung der Landesregierung wird voraussichtlich positiv ausfallen. Nun ist der Zeitpunkt, die bisher angewandten Kommunikationsstrategien kritisch zu hinterfragen und weiter zu entwickeln. Das vergangene Jahr hat gezeigt, dass trinationale und dreisprachige Kommunikation nicht nur einen vermehrten Übersetzungsaufwand mit sich bringt.

Es ist notwendig, dass wir das regionalpolitische Vorgehen der drei Nationen besser verstehen lernen. Pragmatisches, am sogenannten Poldermodell orientiertes Handeln (Niederlande), individualisierte Steuerung (Belgien) und planorientierte Entwicklung (Deutschland) gehen nicht immer überein. Auch inhaltlich kommt es durchaus zu Missverständnissen: Während Nachhaltigkeit auf niederländischer Seite bedeuten kann, ein Gebäude abzureißen und eventuell wiederzuverwerten, wird mit diesem Begriff in der deutschen Teilregion vielleicht eher eine Form des romantischen Naturschutzes verknüpft. Und die belgische Seite hat sich bis dato wenig mit den Strategien nachhaltiger Entwicklung auseinandergesetzt.

Ferner müssen auf der jeweils anderen Seite eigene „Türöffner“ gefunden werden, die sich gemeinsam mit uns für eine grenzüberschreitende Regionalentwicklung einsetzen. Denn nicht nur das Verwaltungs- und Hierarchieverständnis ist unterschiedlich, auch die regionalen Träger von außerstaatlicher Entwicklung finden sich in anderen Netzwerken und Verbänden.

Darüber hinaus wurde deutlich, dass die Arbeit mit Initiativnehmern vor Ort, die persönlichen und Gruppengespräche eine wesentlich höhere Dynamik entwickeln als groß angelegte Werkstätten. Hier lernen sich die Akteure kennen und es wird die wichtigste Basis für grenzüberschreitende Kooperationen geschaffen: Vertrauen.

Die Erfahrungen der letzten eineinhalb Jahre lassen vor diesem Hintergrund auf erste Empfehlungen für ein trinationales Regionalmanagement schließen:

- Gemeinsame grenzuiberschreitende Denk- und Diskursräume als Grundlage für trinationales Handeln sind eminent wichtig, derzeit jedoch noch Mangelware.

- Planungspraktisches und kulturelles Wissen kann nicht innerhalb kürzester Zeit angeeignet werden. Beziehungsnetzwerke müssen in allen drei Ländern verankert sein. Idealerweise ist das Management also trinational besetzt.

- Es bedarf einer hohen Flexibilität bei der Wahl der Strategie: Was in Deutschland logisch erscheinen mag, kann in Belgien auf Unverständnis stoßen.

Von Anfang an sollte immer wieder herausgestellt und reflektiert werden, welchen Nutzen die Partner von grenzüberschreitender Zusammenarbeit haben. Je konkreter dieser formuliert werden kann, desto leichter ist der beträchtliche Mehraufwand zu begründen, der Kommunikation über Sprach- und Staatsgrenzen hinweg unvermeidbar mit sich bringt.

Und vor allem gilt es deutlich zu machen, dass die Möglichkeit, von den Nachbarn zu lernen ein ungeheures inhaltliches und strategisches Pfund ist, mit dem wir wuchern können. Denn schließlich heißt unser zentrales Motto nicht umsonst Viv(r) e la différence (Lebe den Unterschied - Es lebe der Unterschied)!

\section{Die Autorlnnen}

Frauke Burgdorff und Ralf P. Meyer arbeiten als wissenschaftliche Mitarbeiterin bzw. als Leiter der Geschäftsstelle bei der EuRegionale 2008.

Kontakt: EuRegionale 2008, Dennewartstr. 25-27, 52068 Aachen. Tel. 0241/ 96310-74/-39, Fax -33, E-mail: info@euregionale2008.de 
(c) 20I0 Authors; licensee IÖW and oekom verlag. This is an article distributed under the terms of the Creative Commons Attribution Non-Commercial No Derivates License (http://creativecommons.org/licenses/by-nc-nd/3.o/), which permits unrestricted use, distribution, and reproduction in any medium, provided the original work is properly cited. 